Aristizabal, P., Rodríguez-Fernández, A. Rodríguez-Miñambres, P \& Fernández-Zabala, A. (2015). El desarrollo de las competencias transversales en segundo curso de los Grados de Educación Infantil y Primaria. Revista Electrónica Interuniversitaria de Formación del Profesorado, 18 (3), 25-37.

\title{
El desarrollo de las competencias transversales en segundo curso de los Grados de Educación Infantil y Primaria
}

\author{
Pilar Aristizabal ${ }^{*}$, Arantzazu Rodríguez-Fernández, Paloma Rodríguez-Miñambres, Arantza \\ Fernández-Zabala \\ Universidad del País Vasco / Euskal Herriko Unibertsitatea
}

\section{Resumen}

Para dar respuesta al desarrollo de las competencias transversales, desde los Grados de Educación de la Escuela de Magisterio de Vitoria-Gasteiz (EMVG) se han implementado trabajos interdisciplinares de módulo innovadores basados en metodologías activas. En este trabajo se presenta una experiencia de Aprendizaje por Proyectos y Análisis de Caso, relativa al $2^{\circ}$ semestre del $2^{\circ}$ curso. En total, 171 estudiantes cumplimentaron el cuestionario de satisfacción con la nueva metodología. Los resultados indican que el alumnado está satisfecho con el sistema del trabajo interdisciplinar y que han conseguido desarrollar dichas competencias. No obstante, se perciben algunas áreas sobre las que incidir.

\section{Palabras clave}

Competencias transversales; trabajo interdisciplinar; módulo; aprendizaje por proyectos; innovación educativa.

\section{Development of cross competences in second course of Childhood and Elementary Education Degrees}

\begin{abstract}
In order to give an answer to the development of cross-disciplinary skills, an innovative methodology has been implemented in the Education Degrees at the Teacher Training School of Vitoria-Gasteiz: module interdisciplinary work. This paper presents an experience on Project Learning and a case analysis that took place in the second semester of the second year at university. 171 students completed the questionnaire to assess satisfaction with the new methodology. Results indicate that students are satisfied with the


interdisciplinary work system and that they have managed to develop those skills. Nevertheless, some areas are to be considered and focused on for improvement.

\section{Key words}

Cross-disciplinary competences; interdisciplinary work; module; project learning; educational innovation.

\section{Introducción}

\section{El desarrollo de competencias transversales y el sistema modular}

Actualmente la universidad se encuentra inmersa en el proceso de convergencia para la creación de un espacio de educación superior común en toda Europa. Entre los cuatro pilares centrales sobre los que se asienta el paradigma del Espacio Europeo de Educación Superior (EEES), destaca la adquisición en las distintas titulaciones, de determinadas competencias específicas y genéricas (o también denominadas transversales), que suponen una medida común que facilite la movilidad. Desde los años sesenta se ha venido hablando del concepto de competencia (Spencer, McClelland y Spencer, 1992), siendo definido como “(...) un saber hacer complejo resultado de la integración, movilización y adecuación de capacidades y habilidades (de orden cognitivo, afectivo, psicomotor o social) y de conocimientos, utilizados eficazmente en situaciones que tengan un carácter común" (Lasnier, 2000).

Este proceso de convergencia, ha supuesto también un cambio en la concepción de las metodologías docentes (Sánchez y Zubillaga, 2005). Al formular los planes de estudio tomando como eje rector las competencias y habilidades a adquirir, se obliga al profesorado a incorporar al aula metodologías diferentes a la tradicional clase magistral. Se busca que el alumnado sea capaz de saber hacer, ser y estar, en lugar de sólo saber (Delors, et. al. 1996; Martin-Domínguez, y Lavega, 2013). Para ello, se plantean actividades formativas más constructivistas, más participativas y activas, asegurando la adquisición de conocimientos, actitudes, capacidades y destrezas necesarias para responder adecuadamente a las demandas de su desempeño profesional.

Para promover este cambio metodológico subyacente a la convergencia europea, la Universidad del País Vasco/Euskal Herriko Unibertsitatea (UPV/EHU) ha desarrollado un modelo marco propio de actuación docente denominado IKD (Ikaskuntza Kooperatiboa eta Dinamikoa / Enseñanza-aprendizaje Cooperativo y Dinámico) que se convierte en eje del desarrollo curricular en esta universidad (Alkorta, 2011-2012). Este modelo se apoya en las metodologías activas de aprendizaje, pudiendo cada titulación y centro docente interpretarlo de forma flexible y local. Partiendo del modelo IKD, desde los Grados de Educación, la Escuela de Magisterio de Vitoria-Gasteiz (EMVG) ha apostado por desarrollar las competencias transversales a partir de metodologías activas y en contextos lo más parecidos a la realidad, pues no debe olvidarse que la formación debe ponerse en relación con la complejidad que los y las estudiantes pueden encontrar en su vida profesional. Conviene recordar que una competencia transversal es aquella que se considera como una parte fundamental del perfil profesional y formativo propio de todas o de la mayor parte de las titulaciones, y que incluye una serie de habilidades cognitivas, metacognitivas, conocimientos instrumentales y actitudes (Yániz y Villardón, 2006). La segunda apuesta de la EMVG ha sido adoptar un planteamiento modular, basado en la elaboración de trabajos interdisciplinares, ya que estas competencias deben trabajarse desde diferentes asignaturas al mismo tiempo. Cada semestre se organiza en torno a un módulo, desde el que se aborda un tema concreto sobre el cual el alumnado tendrá que realizar un trabajo de 
carácter interdisciplinar, integrando contenidos aportados por las cinco asignaturas que comparten el semestre.

La intención de estas actividades interdisciplinares de módulo (AIM) es facilitar al alumnado experiencias de aprendizaje integradas, donde diferentes puntos de vista pertenecientes a diferentes campos del saber se integren en un todo alrededor de un tema, problema, caso... central, que será el núcleo alrededor del cual se organizará el aprendizaje. Dado que los ritmos de adquisición de las competencias pueden ser diferentes, se ha planteado un sistema de seguimiento y tutorización del alumnado que consta de una serie de sesiones que son previamente consensuadas por todo el profesorado en las reuniones de coordinación.

La carga de trabajo de cada AIM, es de 5 créditos ECTS, 1 crédito por cada una de las asignaturas implicadas. Cada asignatura destina un $20 \%$ de su nota a la calificación del AIM. Para asegurar una mayor objetividad en la valoración del trabajo, el desarrollo competencial se evalúa mediante una rúbrica que se facilita a todo el alumnado al comienzo del semestre, de forma que éste pueda saber desde el primer momento qué se le va a pedir y qué se espera de su trabajo. La evaluación se hace de forma interdisciplinar, esto es, la nota final que reciba el grupo por el proyecto realizado, será acordada por el profesorado del módulo.

\section{El módulo de Diversidad en la Escuela}

El principio rector del Marco de Acción de la Conferencia Mundial sobre Necesidades Educativas Especiales: Acceso y Calidad (UNESCO y Ministerio de Educación y Ciencia de España, 1994) sostiene que las escuelas deben acoger a todos los niños y niñas independientemente de sus condiciones personales, culturales o sociales; tengan o no discapacidad, vivan en la calle, sean de minorías étnicas, lingüísticas o culturales, o de zonas desfavorecidas o marginales. De ahí que, el alumnado egresado en los Grados de Educación Infantil y Primaria, deba desarrollar las competencias necesarias para hacer frente al importante reto de atender a la diversidad en la escuela. Por esta razón, el cuarto módulo (segundo semestre del segundo curso) se dedica a trabajar el tema de La diversidad en la escuela, abarcando las siguientes asignaturas: Organización escolar, Tecnologías de la información y la comunicación en educación, Didáctica de la lengua en contextos multilingües, Bases de la escuela inclusiva y Dificultades en el desarrollo y en el aprendizaje.

El trabajo AIM para este módulo consiste en la elaboración de un plan de acogida para alumnado de origen extranjero, un plan de intervención lingüística para el aula y unas pautas de intervención psicoeducativa para un alumno o alumna con necesidades educativas especiales, utilizando los recursos proporcionados por las TIC para publicar sus trabajos.

A partir de este trabajo interdisciplinar se deben adquirir las siguientes dos competencias acordes con la temática del módulo:

- Identificar y comprender las principales variables del proceso y las fases de acogida al alumnado de origen extranjero necesarias para una intervención adecuada en el aula y en el centro a través de un correcto diseño y planificación de las actividades de acogida aplicando diversos recursos didácticos con criterios de inclusión.

- Detectar las necesidades del alumnado y sus dificultades tanto de aprendizaje como de participación en el aula o en el centro, y saber darles respuesta desde un enfoque multidisciplinar a través de una intervención adecuada que favorezca la inclusión. 
La metodología planteada para este AIM se ha basado en el Aprendizaje Basado en Problemas orientado a Proyectos (puesto que el producto final debe ser un proyecto), combinado con un análisis de caso, dado que experiencias anteriores obtienen resultados satisfactorios en la formación de los futuros maestros y maestras (Arias-Gundín, Fidalgo y García, 2008; Davis y Wilcock, 2005; Orejudo, Fernández y Garrido, 2008). A partir del escenario presentado, los y las estudiantes de grado planean y evalúan un proyecto, con un aprendizaje a largo plazo y tareas centradas en el estudiante, en lugar de actividades o lecciones cortas y aparentemente aisladas unas de otras (Challenge 2000 Multimedia Project, 1999). Un planteamiento de enseñanza-aprendizaje mucho más constructivista, puesto que el alumnado aprende mientras va construyendo ideas o conceptos desde sus conocimientos previos (Karlin y Vianni, 2001).

El punto de partida de este AIM sitúa al o la estudiante en una escuela ficticia que recibe una gran cantidad de alumnado extranjero. Aprovechando que próximamente se van a incorporar a la escuela una alumna y dos alumnos de distintas nacionalidades, el centro se ha propuesto desarrollar un Plan de Acogida, junto con un plan de intervención lingüística para el aula. Pero, dado que la niña presenta un trastorno del desarrollo añadido (un trastorno del espectro autista), también es necesario plantear las pautas para una intervención psicoeducativa. El alumnado del módulo Diversidad en la Escuela debe tomar el rol de tutor o tutora de esa clase y desarrollar esas tres tareas.

Dado que es un trabajo de cierta envergadura, deben sacarlo adelante en grupos de cuatro personas y a lo largo de diversas fases cuya ejecución supone los distintos entregables del semestre: a) deben realizar una justificación razonada del tipo de plan de acogida que van a diseñar; b) detectar las fases y niveles de dicho plan de acogida; c) diseñar las actividades dirigidas al alumnado y la familia; y d) entrega del AIM.

En cuanto al análisis de caso que se les pide, los pasos son cuatro: análisis de las dificultades de la alumna con dificultad del desarrollo, comunicación a la familia de lo observado en el aula, análisis de las características propias de la dificultad y diseño de las pautas generales de intervención psicoeducativa que se debería llevar a cabo.

\section{Objetivos de la investigación}

Este trabajo surge de la preocupación y del interés del profesorado del módulo por conocer cómo ha resultado la implementación del trabajo modular. El cometido principal del estudio que aquí se presenta consiste en analizar la opinión del alumnado de Grado en torno a la innovadora implementación del AIM Diversidad en la escuela diseñado para trabajar las competencias transversales de segundo curso. Este objetivo general se concreta en los siguientes tres objetivos específicos: Analizar la valoración general del AIM por parte del alumnado, concretar la opinión del alumnado sobre el sistema de tutorización del mismo y ofrecer diversas perspectivas de mejora.

\section{Metodología}

\section{Participantes}

En la realización del trabajo modular, durante el curso 2011-2012, participaron un total de 209 estudiantes de segundo curso pertenecientes a los Estudios de Grado de Magisterio. Cumplimentaron el Cuestionario de Satisfacción con el módulo, 171 estudiantes, 76 de Educación Infantil y 95 de Educación Primaria.

Instrumentos de recogida de información 
La satisfacción con el trabajo interdisciplinar fue medida a través de un cuestionario ad hoc ya que este estudio trata de conocer las opiniones del alumnado en torno a la realización de trabajos interdisciplinares para el desarrollo de las competencias transversales (Buendía, Colás, y Hernández, 1998).

Dicho cuestionario está formado por 19 ítems orientados a conocer la percepción del alumnado en torno a la implementación del módulo. Para este trabajo se han seleccionado las respuestas dadas a los ítems centrados en el análisis del trabajo modular. Concretamente, se analizan las respuestas dadas a las preguntas $n^{\circ} 15$ (En tu opinión, ¿en qué medida se han desarrollado las competencias a adquirir en el módulo?), la $n^{\circ} 16$ (Con respecto al trabajo modular ¿qué opinión tienes del mismo?, ¿se han conseguido las competencias planteadas?) $n^{\circ} 17$ (En tu opinión, ¿qué es lo que se ha hecho bien en este módulo o cuatrimestre?), y $n^{\circ} 18$ (¿Qué se podría mejorar?). En cuanto al formato de respuesta de los ítems es variado. Así, mientras que la pregunta $\mathrm{n}^{\circ} 15$ es una escala tipo Likert con cinco opciones (donde $1=$ muy poco y $5=$ mucho), las otras tres preguntas presentan un formato de respuesta abierto, dado que permite recoger información rica en matices (Bisquerra, 2004).

\section{Procedimiento}

El cuestionario fue cumplimentado de manera individual por las y los estudiantes en horario lectivo y en presencia de profesorado implicado en el trabajo modular. El tiempo de aplicación del mismo fue de 20 minutos. Se aseguró a todas las personas participantes el anonimato con el fin de favorecer la sinceridad en sus respuestas y de prevenir, de este modo, la probabilidad del efecto de deseabilidad social.

La metodología empleada presenta una combinación entre la metodología cuantitativa y la cualitativa. En cuanto al análisis de la información cuantitativa referida a la pregunta $n^{\circ} 15$ se ha llevado a cabo mediante el paquete estadístico SPSS versión 20 para Windows. Respecto al análisis de la información cualitativa recogida a través de las preguntas $n^{\circ} 16, n^{\circ} 17$ y $n^{\circ} 18$, se ha realizado una clasificación temática de las ideas expresadas, elaborando así un sistema categorial que ha ayudado a estructurar y ordenar las respuestas obtenidas.

\section{Resultados}

Teniendo en cuenta los objetivos de este estudio, a continuación se presentan los resultados más destacados agrupados en tres grandes bloques: 1) valoración general que hace el alumnado en torno al trabajo interdisciplinar planteado en este módulo; 2) su opinión sobre el sistema de tutorías puesto en marcha en dicho módulo; 3) sugerencias de mejora.

\section{Valoración general del trabajo modular}

La valoración general sobre el trabajo interdisciplinar realizada por el alumnado giró en torno a los siguientes temas: organización general del trabajo, temática elegida para el trabajo, nivel de exigencia del mismo, y desarrollo y consecución de las competencias transversales.

En cuanto a la organización del trabajo, los y las estudiantes destacan que está bien planteado y que, si en el futuro profesional se encuentran en una situación similar a la descrita en el caso, sabrán cómo actuar.

"En mi opinión el trabajo está bien planteado ya que si en el futuro nos encontramos en una situación así, sabremos cómo abordarla”. 
Valoran bien la guía que se les proporciona al inicio del semestre y la distribución del trabajo a lo largo del mismo. Opinan que se les dio abundante información para la realización del trabajo y que estaban claras las fuentes que debían utilizar:

"La distribución del trabajo. Esto es, no hemos tenido que andar venga trabajar en el último momento. Lo que había que hacer estaba bien explicado. La guía del módulo estaba bien hecha".

"Estaba muy claro de dónde teníamos que conseguir la información y teníamos mucha información para hacer el trabajo".

Otro aspecto que destacan como positivo es que el trabajo les exige funcionar con cierta autonomía:

"Me parece positivo porque hemos tenido libertad para organizar el trabajo como queríamos".

Sin embargo, critican que, según van pasando las semanas, el profesorado les va dando pautas sobre cosas que deberían incluir en el trabajo, cómo lo deberían hacer etc. En este sentido, reivindican que se marquen las pautas claramente desde el principio. Critican también que ha habido grupos en los que algún profesor o profesora no ha participado en el módulo. Consideran que es injusto que en esos grupos no se haya exigido la participación de dicho profesorado:

"No ha habido igualdad entre todas las clases. El alumnado del otro grupo no ha tenido que hacer todo el trabajo, su carga ha sido menor y eso no me parece justo".

Se quejan también de la falta de coordinación entre el profesorado, de que la información se les da tarde y siempre hay cambios de última hora:

"Ha habido falta de coordinación y cambios de última hora que dificultan la realización del trabajo".

A pesar de ello, han percibido en el profesorado un esfuerzo por coordinarse, aunque opinan que no siempre se ha conseguido.

"El profesorado ha invertido muchas horas en reuniones de coordinación y yo valoro ese esfuerzo".

Señalan asimismo, la implicación de algunas profesoras y profesores del módulo y la ayuda recibida por parte de estas personas a la hora de resolver dudas y brindar apoyo a lo largo de todo el proceso. Consideran que los contenidos de muchas de las asignaturas han sido útiles de cara a la elaboración del trabajo. Asimismo les ha parecido muy interesante la utilización de una wiki como soporte digital para el trabajo interdisciplinar.

En relación a la temática elegida para el trabajo -diseño de un plan de acogida para un centro con gran diversidad cultural y una intervención lingüística y psicoeducativa en un aula de Educación Infantil o Educación Primaria- ha sido muy bien valorada. Resaltan que han aprendido muchas cosas nuevas y que puede ser muy útil para su futuro profesional. Subrayan que les ha servido para reflexionar sobre el tema de la interculturalidad y para concienciarse en torno a las situaciones de discriminación que puede haber en la escuela, así como sobre el significado del término inclusión educativa. Finalmente apuntan que les ha servido tanto a nivel personal como profesional. Todo lo anterior se recoge en estas voces:

"El tema me parece interesante y totalmente necesario de abordar. Ha sido muy interesante tanto a nivel personal como profesional". 
"El trabajo modular es un trabajo interesante en el que se tratan temas que hacen referencia a la realidad social".

"Lo que hemos aprendido nos será útil en el futuro, para trabajar como profesoras"

El nivel de exigencia del trabajo modular, en general, les ha parecido excesivo para el tiempo que se le dedica en las diferentes asignaturas. Opinan que ha sido demasiado complejo, sobre todo teniendo en cuenta que va dirigido a alumnado de segundo curso. Además, subrayan que el nivel de exigencia varía en función del profesor o profesora que tutoriza el trabajo, lo cual no les parece justo. Critican que, en algunas asignaturas, los contenidos relacionados con el trabajo modular no se trabajan hasta el final del semestre dificultando su incorporación al trabajo. Las siguientes voces resumen lo dicho en este apartado:

"La carga de trabajo me ha parecido excesiva para alumnos y alumnas de $2^{\circ}$ curso".

"El nivel de exigencia ha sido diferente, dependiendo del profesor o profesora".

"Algunos profesores o profesoras no han trabajado el tema hasta los últimos días".

En cuanto al desarrollo de las competencias transversales los datos se muestran en la tabla 1.

Tabla 1.

Percepción del alumnado sobre el desarrollo de las competencias

\begin{tabular}{|l|c|c|c|c|c|}
\hline Competencias & $\begin{array}{c}\text { Muy } \\
\text { Poco (1) }\end{array}$ & $\begin{array}{c}\text { Poco } \\
\text { (2) }\end{array}$ & $\begin{array}{c}\text { Suficiente } \\
\text { (3) }\end{array}$ & $\begin{array}{c}\text { Bastante } \\
\text { (4) }\end{array}$ & $\begin{array}{c}\text { Much } \\
\text { o (5) }\end{array}$ \\
\hline $\begin{array}{l}\text { 1. Identificar y comprender las principales } \\
\text { variables del proceso y las fases de } \\
\text { acogida al alumnado de origen extranjero } \\
\text { necesarias para una intervención } \\
\text { adecuada en el aula y en el centro a través } \\
\text { de un correcto diseño y planificación de } \\
\text { las actividades de acogida aplicando } \\
\text { diversos recursos didácticos con criterios } \\
\text { de inclusión }\end{array}$ & $3,77 \%$ & $15,09 \%$ & $30,19 \%$ & $47,17 \%$ & $3,77 \%$ \\
\hline $\begin{array}{l}\text { 2. Detectar las necesidades del alumnado } \\
\text { y sus dificultades tanto de aprendizaje } \\
\text { como de participación en el aula o en el } \\
\text { centro, y saber darles respuesta desde un } \\
\text { enfoque multidisciplinar a través de una } \\
\text { intervención adecuada que favorezca la } \\
\text { inclusión. }\end{array}$ & $5.88 \%$ & $11.76 \%$ & $35.29 \%$ & $43.14 \%$ & $3.92 \%$ \\
\hline
\end{tabular}

Respecto a la primera competencia a adquirir en el módulo, se puede destacar que un 81, $13 \%$ del alumnado considera que se ha desarrollado en mayor o menor grado. Así, mientras que $30,19 \%$ opinan que se ha desarrollado de forma suficiente, el 50,94\% opina que se ha desarrollado bastante o mucho. Sin embargo, hay un 18,86\% que considera que se ha desarrollado poco o muy poco. Aspecto este que tendrá que ser considerado cursos posteriores. En cuanto al desarrollo de la segunda competencia, los resultados obtenidos son ligeramente más altos que en la primera. Se puede observar que un $82,35 \%$ alumnado considera que ha adquirido esta segunda competencia en mayor o menor grado. Así, el $35,29 \%$ opina que se ha desarrollado de forma suficiente, el $47,06 \%$ opina que se ha 
desarrollado bastante o mucho. No obstante, en este caso también, nos encontramos con que hay un $17,64 \%$ que considera que se ha desarrollado poco o muy poco esta segunda competencia.

En la valoración cualitativa sobre la consecución de las competencias del trabajo, el alumnado afirma que han sido cumplidos y que el trabajo planteado les ha servido para, además de conocer lo que es un Plan de Acogida, ser capaces de elaborarlo y, en su caso, de actuar ante una situación como la que se les propone en el trabajo interdisciplinar. Sin embargo, matizan que les ha costado un gran esfuerzo y que, si lo han logrado, ha sido por lo que han trabajado fuera de la universidad, por su cuenta:

"Se han cumplido, pero en mi opinión, se han podido cumplir sobre todo gracias al trabajo que hemos hecho nosotras fuera de aquí y a la colaboración entre compañeros y compañeras".

En el colectivo de los que piensan que no se han cumplido, argumentan que, en su grupo, se han dividido el trabajo y no han conseguido tener una visión de conjunto del mismo.

"No se han cumplido. Nos hemos dividido el trabajo para acabar a tiempo por lo que cada una ha trabajado una parte específica".

\section{Opinión del alumnado acerca del sistema de tutorías}

Las opiniones del alumnado en cuanto al sistema de tutorización del trabajo modular han estado muy divididas. Entre las opiniones favorables, valoran el haber podido trabajar en grupos pequeños y destacan que las sesiones de tutoría han sido importantes para definir cuáles son los ejes del trabajo y orientar adecuadamente el mismo, así como para resolver dudas. También les han ayudado a comprender mejor las diferentes fases del trabajo y a mejorar lo realizado con las sugerencias del tutor o tutora $y$, en definitiva, han permitido hacer un seguimiento más individualizado del trabajo.

"Han sido muy útiles, nuestra tutora nos ha dejado claro lo que se nos pide y nos ha dado orientaciones para mejorar el trabajo".

Sin embargo, como apuntábamos al principio, no han faltado las voces críticas. En algunos casos, dicen que las tutorías han sido muy pobres y que han funcionado mejor o peor, dependiendo del tutor o tutora que les ha tocado. Se quejan de que todo el profesorado no tiene clara la finalidad del trabajo ni los pasos a seguir, por lo que la información proporcionada no ha sido clara:

"Sí, me han resultado muy útiles porque han servido para responder dudas pero esto no ha sido así en todos los casos, depende del tutor o tutora".

"Las tutorías no valen para nada. Es algo que el profesorado tiene que hacer, pero en mi opinión, no se identifican con ese modo de trabajar".

"Parece que ni los tutores tienen claro que hay que hacer en el trabajo, no dan una información clara".

También creen que son más útiles las tutorías habituales de cada profesor o profesora, ya que es ahí donde verdaderamente reciben la ayuda necesaria.

"Los últimos días hemos ido a tutorías y es ahí donde hemos recibido mayor ayuda, las tutorizaciones de módulo no nos han servido".

Reclaman que en las tutorías se deberían dar criterios más concretos y un esquema claro de trabajo. 
"No son demasiado útiles. En mi opinión, nos tendrían que dar criterios más claros para elaborar el trabajo. No hemos tenido un esquema claro".

Por último, a pesar de todas las críticas anteriores, destacan que el profesorado ha tenido una buena predisposición para ayudar en la elaboración del trabajo y que sin la ayuda de las tutorías se hubieran sentido perdidas. Esto queda recogido en la siguiente voz:

"Si no hubiese habido tutorías, nosotras hubiéramos estado totalmente perdidas".

\section{Sugerencias de mejora del trabajo modular}

Las respuestas recogidas en este apartado giran en torno a tres ejes: la organización del trabajo, las orientaciones para la realización del mismo y las sesiones de tutorización.

En cuanto a la organización del trabajo, reivindican que tiene que haber mayor coordinación y una mejor comunicación entre el profesorado, de modo que se dé la información de la manera más parecida posible por parte de todo el profesorado.

"Debería mejorar la comunicación entre el profesorado. Que no se explique el trabajo al final".

"Pediría al profesorado que se pongan de acuerdo, que tengan las cosas claras, de lo contrario, nunca nos aclaramos con las explicaciones que nos dan".

Un grupo más de propuestas relacionadas con el contenido del trabajo: que el modulo tenga un fin más concreto y que no tenga una extensión tan grande.

"Propondría que el modulo tuviera un fin más concreto, como en el caso del tríptico del año pasado, para no tener una extensión tan grande".

En cuanto a la manera de llevar a cabo el trabajo de módulo, piden que se deje más tiempo en las horas de clase y se ofrezca la ayuda necesaria para ello.

"Que se deje tiempo en clase para hacer el trabajo modular ya que estando el profesor o profesora en clase habría posibilidad de aclarar las dudas en el momento y así tendríamos mayor seguridad en cuanto a la adecuación del trabajo".

Por otro lado, plantean la posibilidad de que puedan acudir representantes de aula a las reuniones de coordinación.

"Que en las reuniones de coordinación puedan acudir representantes de aula para expresar sus dudas, ideas o sugerencias y conocer mejor la organización entre el profesorado, la información dada al alumnado...Que se escuchen nuestras propuestas y se lleven adelante".

Plantean que la participación en el módulo, debería ser obligatoria para el profesorado ya que así se aseguraría que en todos los grupos tienen que hacer el mismo trabajo.

"Me parece imprescindible que en todas las clases haya que hacer el mismo trabajo. Que no se haga una parte porque un profesor no quiere participar, no me parece normal".

En relación a las orientaciones para la realización del trabajo, insisten en que la información necesaria para hacer el trabajo, así como los temas que hagan referencia al módulo, tendrían que darse al comienzo del semestre en cada asignatura para tener tiempo de ir haciendo el trabajo a lo largo del mismo.

"Mejoras: - proporcionar información desde el principio. - que todos los tutores den la misma información. - los temas que hagan referencia al módulo tratarlos al principio del semestre". 
También solicitan que se les dé una estructura mínima de lo que tiene que ser el trabajo y que se facilite un esquema básico con el que empezar a desarrollar el informe escrito. Otra propuesta que hacen es, que si no se pudiera anticipar esa información, se proporcionase la bibliografía necesaria para poder ir adelantando la redacción.

Asimismo proponen que para evitar contradicciones en la información proporcionada por el profesorado, se den explicaciones comunes a todo el alumnado en las horas de clase.

"Propondría que el profesorado de las diferentes materias dé explicaciones comunes en las horas de clase".

En definitiva, nos piden una mayor implicación y orientación a la hora de hacer el trabajo tal y como se recoge en esta voz:

“... pero tenemos que percibir más organización e implicación. No digo que se nos tengan que proporcionar todos los contenidos y materiales que hay que incluir en el trabajo, no, pido que se nos guíe más".

En cuanto a las sesiones de tutorización, para reducir las diferencias en las mismas, proponen que el profesorado debería elaborar unos guiones de trabajo comunes con los puntos a abordar en cada sesión. También hay algunas propuestas concretas interesantes, como por ejemplo, que se organicen tutorizaciones conjuntas o que haya rotación de tutores y tutoras por los diferentes grupos para resolver las dudas de cada asignatura sin necesidad de recurrir a las tutorías convencionales. Este sistema tendría la ventaja de que podrían escucharse las dudas de los demás compañeros y compañeras de clase.

\section{Discusión y conclusiones}

Las personas que suscribimos este artículo, partimos de la convicción de que trabajar por proyectos o análisis de casos es una buena manera de responder a las necesidades y los retos que tendrán que afrontar las y los futuros docentes y de garantizar la consecución de las competencias transversales.

Se ha procurado abordar dichas competencias transversales con un enfoque didáctico, cercano a la realidad multicultural de las aulas que nuestro alumnado verá en su quehacer profesional. Que el trabajo resulte motivador, verosímil y eficaz de cara a la obtención de las competencias y que el alumnado vea su aplicación en el mundo real, más allá del aula (Blank, 1997; Dickinson, et al, 1998; Harwell, 1997). Y que, al desempeñar un papel activo en todo el proceso encuentren los proyectos motivadores, divertidos y retadores (Challenge 2000 Multimedia Project, 1999; Katz, 1994).

Somos conscientes, de que cuando se trata de innovación educativa, trabajar conjuntamente desde varias áreas de conocimiento supone un gran compromiso por parte del profesorado universitario por aunar esfuerzos en la misma dirección y compartir una misma visión del trabajo a presentar al alumnado, compromiso que no todo el profesorado está dispuesto a asumir.

Tampoco todo el alumnado responde positivamente a este tipo de trabajos, esto concuerda con estudios anteriores realizados en torno a las actitudes del alumnado en los trabajos por proyectos que requieren un alto grado de responsabilidad e implicación (Mérida, 2007).

Las conclusiones del estudio no dejan lugar a dudas sobre qué valora bien el alumnado. En primer lugar, el tema planteado en el trabajo interdisciplinar les resulta muy atrayente para su futuro profesional; en segundo lugar, valoran muy positivamente el dejar tiempo de las asignaturas en clase para avanzar en el trabajo modular, así como que desde el inicio de 
curso haya una guía docente que explicita las fases del trabajo, el cronograma de entregables de cada asignatura y las referencias que deben consultar para realizar el informe escrito.

En cuanto a los aspectos que juzgan negativos destacan dos líneas; por un lado, se muestran críticos con el profesorado aludiendo a la falta de implicación y a los problemas de coordinación entre algunos docentes del módulo; por otro lado, se quejan de que tienen que dedicar demasiado tiempo a la realización del trabajo en comparación con el valor final en la nota ( 2 puntos) y de que, en algunas asignaturas, reciben tarde el material que necesitan, en las dos últimas semanas del semestre.

Esto revela la resistencia del alumnado a cambiar de manera de pensar y actuar. En este sentido, alumnos y alumnas, deberían implicarse en estas metodologías novedosas que les posibilitan conseguir el desarrollo de las competencias transversales, entendiendo las dificultades como un estímulo para hacerlo mejor, de tal manera que sean personas más autónomas, participativas y activas en su aprendizaje.

Concluimos, que tanto el profesorado como el alumnado universitario, tienen que llevar a cabo un proceso autoreflexivo sobre sus prácticas, vinculando el proceso formador con el desarrollo de las competencias transversales para comprender mejor la realidad social en la que están inmersos. El proceso de cambio que se ha dado en la cultura pedagógica desde la implantación del EEES conlleva la necesidad de asumir el compromiso con la innovación. Nuestra tarea como docentes universitarios, además de detectar las dificultades y las dudas que puedan surgir en el proceso, es tener una fuerte implicación e interés por cambiar y mejorar (Margalef, 2011). Si somos capaces de esforzarnos en la coordinación entre asignaturas y creemos en lo que estamos haciendo, ese esfuerzo será valorado y reconocido por nuestro alumnado de grado.

\section{Bibliografía}

Alkorta, I. (2011-2012). IKD, un modelo para la educación superior en el siglo XXI = IKD, teaching \& learning Higher Education model for the XXIst Century. Fabrikart: arte, tecnología, industria, sociedad, 10, 14-25.

Arias-Gundín, O., Fidalgo, R., y García, J. N. (2008). El desarrollo de las competencias transversales en magisterio mediante el aprendizaje basado en problemas y el método de caso. Revista de Investigación Educativa, 26(2), 431-444.

Bisquerra, R. (2004). Metodología en la investigación cualitativa. Madrid: La Muralla

Blank, W. (1997). Authentic instruction. En W. E. Blank, y S. Harwell (Eds.), Promising practices for connecting high school to the real world (pp. 15-21). Tampa, Florida: University of South Florida.

Buendía, L., Colás, P., y Hernández, F. (1998). Métodos de investigación en Psicopedagogía. Madrid: McGraw-Hill.

Challenge 2000 Multimedia Project. (1999). Why do projectbased learning? San Mateo, CA: San Mateo County Office of Education.

Davis, C., y Wilcock, E. (2005). Developing, implementing and evaluating case studies in materials science. European Journal of Engineering Education, 30 (1), 59-69.

Delors, J. et al. (1996). La Educación encierra un tesoro. Madrid: Santillana, Ediciones UNESCO. 
Dickinson, K. P., Soukamneuth, S., Yu, H.C., Kimball, M., D’Amico, R., Perry, R., et al. (1998). Providing educational services in the Summer Youth Employment and Training Program [Technical assistance guide]. Washington, DC: U.S. Department of Labor, Office of Policy \& Research.

Harwell, S. (1997). Project-based learning. En W. E. Blank, y S. Harwell (Eds.), Promising practices for connecting high school to the real world (pp. 23-28). Tampa, Florida: University of South Florida.

Karlin, M., y Viani, N. (2001). Project-based learning. Medford, OR: Jackson Education Service District.

Katz, L. G. (1994). The project approach. Urbana: ERIC Clearinghouse on Elementary and Early Childhood Education.

Lasnier, F. (2000). Réussir la formation par competences. Montreal: Guérin.

Margalef, L. (2011). Los encuentros de innovación en docencia universitaria: un entorno para el aprendizaje colaborativo del profesorado y el alumnado. Pulso, (34),11-28.

Martin-Domínguez, J., y Lavega, P. (2013). Enseñar competencias en el Espacio Europeo de Educación Superior. Revista Electrónica Interuniversitaria de Formación del Profesorado, 16(1), 1-4.

Mérida, R. (2007). Hacia la Convergencia Europea: los Proyectos de Trabajo en la docencia universitaria. Electronic Journal of Research in Educational Psychology, 5(13), 825-852.

Orejudo, S., Fernández, T., y Garrido, M. A. (2008). Elaboración y trabajo con casos y otras metodologías activas. Cuatro experiencias de un grupo de profesores de la Facultad de Educación de Zaragoza. Revista Interuniversitaria de Formación del Profesorado, 63(22,3), 21-45.

Sánchez, P., y Zubillaga, A. (2005). Las universidades españolas ante el proceso de convergencia europeo: Análisis de las medidas institucionales y acciones de aplicación y coordinación. Revista de Educación, 337, 169-187.

Spencer, L. M., McClelland, D. C., y Spencer, S. M. (1992). Competency assessment methods; history and state of the art. New York: Hay/McBer Research Press.

UNESCO y Ministerio de Educación y Ciencia de España (1994). Declaración de Salamanca y marco de acción para las necesidades educativas especiales. Salamanca: UNESCO.

Yáñiz, C., y Villardón, L. (2006). Planificar desde competencias para promover el aprendizaje. El reto de la sociedad del conocimiento para el profesorado universitario. Bilbao: Cuadernos Monográficos del ICE, 12. 


\section{Autoras}

Pilar Aristizabal Llorente

Doctora en Pedagogía y Profesora del Departamento de Didáctica y Organización Escolar de la Universidad del País Vasco Vasco / Euskal Herriko Unibertsitatea desde el año 2001. Imparte docencia en la E.U. de Magisterio de Vitoria-Gasteiz en la titulación de Grado de Educación Infantil. Ha publicado algunos artículos en diferentes revistas científicas sobre temas relacionados con la educación, las TIC, el género y la formación del profesorado.

Arantzazu Rodríguez Fernández

Doctora en Psicología y profesora contratada en la Universidad del País Vasco / Euskal Herriko Unibertsitatea. Imparte docencia en la E.U. de Magisterio de Vitoria-Gasteiz en la titulación de Grado de Educación Primaria, concretamente en el módulo de Diversidad en la Escuela, del cual es coordinadora. Ha publicado diversos artículos en revistas nacionales e internacionales de impacto en temas relacionados con la educación y el ajuste escolar.

Paloma Rodriguez-Miñambres

Licenciada en Filología Vasca por la Universidad de Deusto y ha realizado el Master de Psicodidáctica de la Universidad del País Vasco. Actualmente trabaja en la Escuela Universitaria del Profesorado de Vitoria-Gasteiz en el departamento de Didáctica de la Lengua y la Literatura.

Arantza Fernández Zabala

Doctora en Psicología por la Universidad del País Vasco (UPV-EHU) y profesora adjunta del Departamento de Psicología Evolutiva y de la Educación en el campus de Álava. Actualmente es coordinadora del módulo Diversidad en la Escuela en la titulación de Educación Infantil, y se está formando a través del programa "Eragin" en metodologías activas y cooperativas. Su actividad investigadora se ha centrado en la temática del autoconcepto habiendo publicado algunos artículos en diferentes revistas científicas. 